\title{
ON A CLASS OF NODAL NONCOMMUTATIVE JORDAN ALGEBRAS
}

\author{
BY \\ JERRY I. GOLDMAN $\left({ }^{1}\right)$
}

1. A finite-dimensional power-associative algebra $A$ with unity element 1 over a field $F$ is a nodal algebra [6] if each element $x$ of $A$ may be written $x=\alpha 1+z$, where $\alpha$ is in $F$ and $z$ is nilpotent, and if $A$ is not of the form $A=F 1+N$ where $N$ is a nil subalgebra of $A$. The class $K$, of $p^{s}$-dimensional nodal noncommutative Jordan algebras we will consider in this paper is described as follows: let

$$
B_{n}=F\left[1, x_{1}, \ldots, x_{n}\right] \text { with } x_{i}^{p}=0, x_{i}^{0}=1 \text {, for } i=1, \ldots, n,
$$

be a commutative associative truncated polynomial algebra over the field $F$ of characteristic $p \neq 2$. An algebra $A$ in $K$ of dimension $p^{n}$ is taken to be the same vector space as $B_{n}$ but with multiplication given by the product

$$
f g=f \cdot g+\sum_{i, j=1}^{n} \frac{\partial f}{\partial x_{i}} \cdot \frac{\partial g}{\partial x_{j}} \cdot c_{i j},
$$

where $f \cdot g$ is the product of $f=f\left(x_{1}, \ldots, x_{n}\right)$ and $g=g\left(x_{1}, \ldots, x_{n}\right)$ in $B_{n}$ and the $c_{i j}=\frac{1}{2}\left[x_{i}, x_{j}\right]=\frac{1}{2}\left(x_{i} x_{j}-x_{j} x_{i}\right)$ are arbitrary except for the proviso that at least one of them is nonsingular. That is, there must exist a $c_{i j}=\alpha_{i j} 1+w_{i j}$ with $\alpha_{i j} \neq 0$. This implies that $n \geqq 2$.

The class $K$ was constructed by L. Kokoris who proved [2], [3] that every simple nodal noncommutative Jordan algebra is in $K$. He also proved that not all the algebras of the class $K$ are simple.

Two papers have appeared which were concerned with studying derivations of some of the algebras in $K$. The first of these by $\mathrm{R}$. Schafer [7] described derivation algebras for the cases $c_{i j}$ in $F$ and $n=2$ and demonstrated relationships between certain ideals of these algebras and types of simple Lie algebras of characteristic $p$. He made use of two properties which were later generalized by R. Oehmke, namely that the algebras in $K$ for the cases $c_{i j}$ in $F$ and $n=2$ are Lie-admissible and that, for $n=2$, the generators $x_{1}$ and $x_{2}$ could be chosen so that $c_{12}=1+\alpha x_{1}^{p-1} \cdot x_{2}^{p-1}$ for $\alpha$ in $F$.

In his paper [4], Oehmke determined the derivation algebras of all simple Lieadmissible algebras $A$ of $K$. He proved that generators could be chosen for these $A$ which satisfy a useful Lie-multiplication table, which is a generalization of

Received by the editors May 13, 1966.

(1) This paper is taken from the author's Ph.D. dissertation written at Illinois Institute of Technology under the direction of Louis A. Kokoris. 
Schafer's relation for the Lie product of two generators. We call this multiplication table an Oehmke multiplication table in this paper.

The theorems in our $\$ \S 2$ and 3, together with [4] show that existence of generators satisfying Oehmke-type multiplication relations is a necessary and sufficient condition for simplicity and Lie-admissibility of an algebra in $K$. The result of $\$ 3$ concerning the Lie-admissibility of algebras of $K$ having an Oehmke multiplication table is applied to the construction of the example of $\$ 4$. This example is one of a simple algebra in $K$ which is not Lie-admissible and which cannot have an Oehmke multiplication table. Thus, the converse to Theorem 1 is not true and something (Lie-admissibility here) must be added to provide a necessary and sufficient condition for simplicity in this context.

The author is grateful to the referee for his suggestions and for pointing out an index error which occurred in equations (2) below in an earlier draft and in [4].

2. We will say that an algebra $A=F 1+F\left[x_{1}, \ldots, x_{n}\right]$ (vector space direct sum) in $K$ has an Oehmke multiplication table (OMT) if the generators $x_{1}, \ldots, x_{n}$ can be chosen such that for $n$ even, $n=2 r$, we have:

$$
\begin{aligned}
{\left[x_{i}, x_{i+r}\right] } & =1+\alpha_{i} x_{i}^{p-1} \cdot x_{i+r}^{p-1} & & \text { for } i=1, \ldots, r, \alpha_{i} \text { in } F, \\
{\left[x_{i}, x_{j}\right] } & =0 & & \text { for } j \neq i+r,
\end{aligned}
$$

and for $n$ odd, $n=2 r+1$, we have (1) holding and

$$
\begin{array}{rlrl}
{\left[x_{2 r+1}, x_{j}\right]} & =0 & & \text { for } j \neq r, 2 r, \\
{\left[x_{2 r+1}, x_{2 r}\right]} & =\alpha x_{r}^{p-1} \cdot\left(1+\beta x_{2 r+1}^{p-1}\right), & \\
{\left[x_{2 r+1}, x_{r}\right]} & =x_{2 r}^{p-1} \cdot\left(1+\beta x_{2 r+1}^{p-1}\right) & & \text { for } \alpha, \beta \text { in } F .
\end{array}
$$

Here, as in the preceding section, we have used the notation, $[x, y]$, for the commutator $x y-y x$ of $x$ and $y$. If we denote the mapping $x \rightarrow[x, y]$ by $D(y)$, i.e., $x D(y)=[x, y]$, then the fact that $D(y)$ is a derivation of $A^{+}$for every $y$ in $A$ is a well-known consequence of the flexible identity.

In [4], Oehmke proved that a simple Lie-admissible algebra in $K$ has an OMT.

We further remark here that $B$ is an ideal of $A$ if and only if $B$ is an ideal of both $A^{+}$and $A^{-}$.

THEOREM 1. Let $A$ be an algebra in K. If $A$ has an OMT, then $A$ is simple.

Proof. Let $B$ be a nonzero ideal of $A$, for $A$ of dimension $p^{n}$. Because of the nature of (1) and (2) we must break up the proof into cases of even and odd numbers of generators.

We first consider an algebra $A$ with an even number, $n=2 r$, of generators for which we can assert the following, due to Oehmke [4, pp. 425-426]. If $m$ is any monomial in $B$ and a generator $x_{i}$ occurs (to any power) in $m$, then $x_{i}$ is in $B$. We reproduce the proof of this claim here, because of typographical errors in [4]. 
Suppose $m=x_{i}^{k} \cdot q$ where $q$ is independent of $x_{i}$. Since $B$ is an ideal of $A^{-}$, [ $m, x_{i+r}$ ] is in $B$. (If $i>r$, Lie multiplication by $x_{i-r}$ will give us the result.) But

$$
\begin{aligned}
{\left[m, x_{i+r}\right] } & =m D\left(x_{i+r}\right)=k x_{i}^{k-1} \cdot x_{i} D\left(x_{i+r}\right) \cdot q+x_{i}^{k} \cdot q D\left(x_{i+r}\right) \\
& =k x_{i}^{k-1} \cdot\left(1+\alpha_{i} x_{i}^{p-1} \cdot x_{i+r}^{p-1}\right) \cdot q=k x_{i}^{k-1} \cdot q \text { if } k>1 .
\end{aligned}
$$

Therefore, we might as well suppose that $k=1$ and that $m=x_{i} \cdot q$ is in $B$.

Using an induction argument and the above method, $m$ may be assumed to be just a product of generators, each with exponent $=1$.

We may further assume that if the generator $x_{k}$ appears in $m$ for $k \leqq r$, then $x_{k+r}$ does not appear. For, if $m=x_{k} \cdot x_{k+r} \cdot s$, then $m D\left(x_{k}\right)=-x_{k} \cdot s$ is in $B$.

The above considerations justify taking $m$ to be of the form $m=x_{i} \cdot x_{j} \cdot \ldots \cdot x_{k} \cdot x_{t}$, where the subscript of each of the distinct generators is less than or equal to $r$.

Hence $m D\left(x_{t+r}\right)=x_{i} \cdot x_{j} \cdot \ldots \cdot x_{k} \cdot x_{t} D\left(x_{t+r}\right)$ is in $B$. But, $B$ an ideal of $A^{+}$and $x_{t} D\left(x_{t+r}\right)$ nunsingular imply that $x_{i} \cdot x_{j} \cdot \ldots \cdot x_{k}$ is in $B$. Continuing this process, we find that $x_{i} \cdot x_{j}$ is in $B$. Thus,

$$
\left[\left(x_{i} \cdot x_{j}\right) D\left(x_{j+r}\right)\right] \cdot\left(x_{j} D\left(x_{j+r}\right)\right)^{-1}=x_{i}
$$

is in $B$, which proves our claim.

Now we introduce a result of A. A. Albert [1, Lemma 21]: the monomial $m=x_{1}^{p-1} \cdot \ldots \cdot x_{2 r}^{p-1}$ is in $B$. By the claim then, $B$ contains every generator, hence $B=A$. This proves Theorem 1 for $n=2 r$.

We now turn to the proof of Theorem 1 for the case of $n=2 r+1$ generators. Using the above quoted result of Albert, the maximal degree monomial

$$
x_{1}^{p-1} \cdot \ldots \cdot x_{2 r+1}^{p-1}
$$

is in $B$. Making use of the multiplication table (1), it is clear that application of the operators $D\left(x_{1}\right), \ldots, D\left(x_{r-1}\right), D\left(x_{r+1}\right), \ldots, D\left(x_{2 r-1}\right)$ to this monomial will, just as in the even case above, guarantee that the monomial

$$
m=x_{1} \cdot \ldots \cdot x_{r-1} \cdot x_{r}^{p-1} \cdot x_{r+1} \cdot \ldots \cdot x_{2 r-1} \cdot x_{2 r}^{p-1} \cdot x_{2 r+1}^{p-1}
$$

is in $B$.

Noting that $x_{k} D\left(x_{1+r}\right)=0$ for $k \neq 1$, we then see that

$$
m D\left(x_{1+r}\right) \cdot\left[x_{1}, x_{1+r}\right]^{-1}=x_{2} \cdot \ldots \cdot x_{r-1} \cdot x_{r}^{p-1} \cdot x_{r+1} \cdot \ldots \cdot x_{2 r-1} \cdot x_{2 r}^{p-1} \cdot x_{2 r+1}^{p-1}
$$

is in $B$. The terms $x_{i}, i=2, \ldots, r-1, r+1, \ldots, 2 r-1$ are eliminated in the same manner, thus showing that $x_{r}^{p-1} \cdot x_{2 r}^{p-1} \cdot x_{2 r+1}^{p-1}$ is in $B$.

The problem has now been reduced to the case of the ideal $B$ containing a maximal degree monomial in the three generators $x_{r}, x_{2 r}$, and $x_{2 r+1}$. The technique for completion of the proof will be easier from the standpoint of both understanding and typography if we illustrate it by just showing that an algebra in $K$ with three generators $x, y, z$ satisfying (1) and (2) is simple. 
Therefore, we will assume it is clear that the methods to come may be carried over to the general case and we will take $A=F 1+F[x, y, z]$ in $K$ satisfying

$$
\begin{aligned}
{[y, x] } & =1+\gamma x^{p-1} \cdot y^{p-1}, \quad \gamma \text { in } F, \\
{[z, y] } & =\alpha x^{p-1} \cdot\left(1+\beta z^{p-1}\right), \\
{[z, x] } & =y^{p-1} \cdot\left(1+\beta z^{p-1}\right),
\end{aligned}
$$

and prove that such an algebra $A$ is simple.

We have $x^{p-1} \cdot y^{p-1} \cdot z^{p-1}$ is in $B$. Thus there is an integer $m=$ the minimal exponent such that $x^{p-1} \cdot y^{p-1} \cdot z^{m}$ is in $B$.

Denote the sth iterate of the mapping $D(x)$ by $D^{s}(x)$. It may be verified, by virtue of (3), that the following two equations hold:

$$
\left(x^{i} \cdot y^{j} \cdot z^{k}\right) D^{s}(x)=P(j, s) x^{i} \cdot y^{j-s} \cdot z^{k}
$$

and

$$
\left(x^{i} \cdot y^{j} \cdot z^{k}\right) D^{t}(y)=\delta P(i, t) x^{i-t} \cdot y^{j} \cdot z^{k}
$$

where $i, j>0, k=0,1, \ldots, p-1, \delta= \pm 1$, and $P(h, q)=h ! /(h-q) !$.

It is clear from (4) and (5) that $x^{a} \cdot y^{b} \cdot z^{m}$ is in $B$ for any $a, b$ such that $1 \leqq a, b$ $\leqq p-1$. Operating on $x \cdot y \cdot z^{m}$ by $D(x)$, we have $x \cdot z^{m}$ in $B$.

Suppose that $m \geqq 1$, and consider the calculation:

$$
\begin{aligned}
\left(x \cdot z^{m}\right) D\left(x^{2}\right) & =2 m x^{2} \cdot z^{m-1} \cdot z D(x) \\
& =2 m x^{2} \cdot y^{p-1} \cdot z^{m-1} \quad \text { for } m \geqq 2 \\
& =2 x^{2} \cdot y^{p-1} \cdot z^{m-1}+2 \beta x^{2} \cdot y^{p-1} \cdot z^{p-1} \quad \text { for } m=1 .
\end{aligned}
$$

Since $x^{2} \cdot y^{p-1} \cdot z^{p-1}$ is in $B$, either of the above two equations implies that $x^{2} \cdot y^{p-1} \cdot z^{m-1}$ is in $B$ and hence that $x^{p-1} \cdot y^{p-1} \cdot z^{m-1}$ belongs to $B$ for $m \geqq 1$, contrary to the minimality of $m$. Therefore $m=0$ and $x^{p-1} \cdot y^{p-1}$ is in $B$, thus $x \cdot y$ is in $B$.

Hence the nonsingular element $(x \cdot y) D(x) D(y)=x D(y)$ is in $B$ and $B=A$. Therefore $A$ is simple and the proof of Theorem 1 is complete. The remarks of the introduction yield the following

Corollary. Let $A$ be in $K$ and suppose either all $c_{i j}$ are in $F$ or $n=2 . A$ is simple if and only if $A$ has an OMT.

3. We now examine the Lie-admissibility of algebras $A$ in $K$ having an OMT. The chief tool used in these considerations will be the criteria of $\mathrm{R}$. Schafer [7, p. 318] which we state in the following manner.

An algebra $A$ in $K$ of dimension $p^{n}$ is Lie-admissible if and only if

$$
\sum_{t=1}^{n} Q_{t}=0
$$


where

$$
Q_{t}=\partial c_{i j} / \partial x_{t} \cdot c_{t k}+\partial c_{j k} / \partial x_{t} \cdot c_{t i}+\partial c_{k i} / \partial x_{t} \cdot c_{t j}
$$

with $2 c_{i j}=\left[x_{i}, x_{j}\right]$ and $1 \leqq i<j<k \leqq n$.

Suppose $A$ is in $K$ and has an even number, $n=2 r$, of generators having an OMT. Since we intend to apply (6) and (7) to this $A$, we examine $Q_{t}$ where we now must have

$$
1 \leqq i<j<k \leqq 2 r .
$$

No generality is lost by assuming $t \leqq r$, since a symmetrical result holds for $t \geqq r+1$.

The only nonzero $c_{t q}$ occurs when $q=t+r$. Suppose that $c_{t k}=c_{t, t+r}$. (Similar reasoning holds for $i=t+r$ and $j=t+r$.) Therefore $c_{t i}=c_{t j}=0$. Hence, from (7), $Q_{t}=\partial c_{i j} / \partial x_{t} \cdot c_{t, t+r}$

But $c_{i j}=0$ unless $j=i+r$ (or $i=j-r$ ). Thus,

$$
Q_{t}=\frac{\partial c_{i, i+r}}{\partial x_{t}} \cdot c_{t, t+r}=\frac{1}{2} \alpha_{i} \frac{\partial\left(x_{i}^{p-1} \cdot x_{i+r}^{p-1}\right)}{\partial x_{t}} \cdot c_{t, t+r}
$$

However, we can assert that $t \neq i$, for otherwise we would have $k=j$, a contradiction, and also we have $t \neq i+r$, for if $t=i+r$, then $k=i+2 r$, which is impossible. (Similarly, $t \neq j$ and $t \neq j-r$.)

Therefore the quantity being differentiated is not a function of $x_{t}$, which implies $Q_{t}=0$. Thus, using (6), $A^{-}$is a Lie algebra and we have proved a portion of the following theorem.

TheOREM 2. Let $A$ be an algebra in K. If $A$ has an OMT, then $A$ is Lie-admissible.

Proof. To deal with the second portion of this theorem, the case of $2 r+1$ generators,

$$
1 \leqq i<j<k \leqq 2 r+1
$$

must hold where we intend to apply (6) and (7). We see that $Q_{t}=0$ for $t \neq r, 2 r$, or $2 r+1$, since for $t$ different from these values the generators concerned satisfy a multiplication of the form (1). The detailed reasoning involves considerations similar to the even case proof of Theorem 2 .

The quantities $Q_{r}, Q_{2 r}$, and $Q_{2 r+1}$ will now be considered separately.

We can exhaust the value possibilities for $Q_{r}$ by considering the four mutually exclusive cases which arise when $k=2 r+1$ and either $i=r$ or $j=r$ or $i \neq r$ and $j \neq r$; or when $k \leqq 2 r$.

Calculations will show that $Q_{r}=0$ in all these cases; we detail some samples of the reasoning for purposes of illustration.

If $k=2 r+1$ and $i=r$, then using (7) we find that

$$
Q_{r}=\partial c_{r j} / \partial x_{r} \cdot c_{r, 2 r+1}+\partial c_{2 r+1, r} / \partial x_{r} \cdot c_{r j} .
$$


Now $c_{2 r+1, r}$ is not a function of $x_{r}$, hence

$$
Q_{r}=\partial c_{r j} / \partial x_{r} \cdot c_{r, 2 r+1} \text {. }
$$

The only nonzero possibility for $Q_{r}$ would be for $j=2 r$, since we cannot have $j=2 r+1=k$. Making the computations for these index values we obtain

$$
\begin{aligned}
Q_{r} & =\frac{\partial}{\partial x_{r}}\left[\frac{1}{2}\left(1+\alpha_{r} x_{r}^{p-1} \cdot x_{2 r}^{p-1}\right)\right] \cdot\left(-\frac{1}{2} x_{2 r}^{p-1} \cdot\left(1+\beta x_{2 r+1}^{p-1}\right)\right) \\
& =\frac{1}{4} \alpha_{r} x_{r}^{p-2} \cdot x_{2 r}^{p-1} \cdot x_{2 r}^{p-1} \cdot\left(1+\beta x_{2 r+1}^{p-1}\right)=0 .
\end{aligned}
$$

As a last example, we consider the case in which $k=2 r+1, i \neq r$, and $j \neq r$. Here we have

$$
Q_{r}=\frac{\partial c_{i j}}{\partial x_{r}} \cdot c_{r, 2 r+1}+\frac{\partial c_{j, 2 r+1}}{\partial x_{r}} \cdot c_{r i}+\frac{\partial c_{2 r+1, i}}{\partial x_{r}} \cdot c_{r j}
$$

Notice that $Q_{r}=0$ for $j=2 r$, since we cannot have $i=2 r=j$ and $i=r$ is disallowed by the case.

For $j<2 r$, the above equation yields

$$
Q_{r}=\partial c_{i j} / \partial x_{r} \cdot c_{r, 2 r+1},
$$

and we note that $c_{i j}$ cannot be a function of $x_{r}$ in these circumstances. Thus $Q_{r}=0$ here.

We can demonstrate that $Q_{2 r}=Q_{2 r+1}=0$ in much the same manner. The cases which exhaust the value possibilities for $Q_{2 r}$ are $k=2 r+1$ and either $i=r$ or $j=r$ or $i \neq r$ and $j \neq r$; or $k \leqq 2 r$. We need only three mutually exclusive cases to represent all the possibilities for $Q_{2 r+1}$. We could have $k=2 r+1$ and either $j=2 r$ or $j<2 r$; or have $k \leqq 2 r$.

The verification that $Q_{2 r+1}=0$ is immediate by inspection in all but the first case: $k=2 r+1$ and $j=2 r$. Here we see

$$
Q_{2 r+1}=\frac{\partial c_{2 r, 2 r+1}}{\partial x_{2 r+1}} \cdot c_{2 r+1, i}+\frac{\partial c_{2 r+1, i}}{\partial x_{2 r+1}} \cdot c_{2 r+1,2 r}
$$

which may admit a nonzero possibility for $i=r$. However, we then find

$$
\begin{aligned}
4 Q_{2 r+1}= & \frac{\partial\left(-\alpha x_{r}^{p-1} \cdot\left(1+\beta x_{2 r+1}^{p-1}\right)\right)}{\partial x_{2 r+1}} \cdot x_{2 r}^{p-1} \cdot\left(1+\beta x_{2 r+1}^{p-1}\right) \\
& +\frac{\partial\left(x_{2 r}^{p-1} \cdot\left(1+\beta x_{2 r+1}^{p-1}\right)\right)}{\partial x_{2 r+1}} \cdot \alpha x_{r}^{p-1} \cdot\left(1+\beta x_{2 r+1}^{p-1}\right) \\
= & \alpha \beta x_{r}^{p-1} \cdot x_{2 r+1}^{p-2} \cdot x_{2 r}^{p-1} \cdot\left(1+\beta x_{2 r+1}^{p-1}\right) \\
& -\alpha \beta x_{2 r}^{p-1} \cdot x_{2 r+1}^{p-2} \cdot x_{r}^{p-1} \cdot\left(1+\beta x_{2 r+1}^{p-1}\right) \\
= & 0 .
\end{aligned}
$$

Therefore $Q_{t}=0$ for $1 \leqq t \leqq 2 r+1$ and Theorem 2 is proved. 
4. We are now in a position to state the next theorem which makes it clear that simplicity alone is insufficient for an OMT.

THEOREM 3. There exist simple algebras of $K$ which are not Lie-admissible and which cannot have an OMT.

Proof. Let $x_{1}, \ldots, x_{2 R}$ for $R>1$, be $2 R$ quantities and let $F$ be a field of characteristic $p \neq 2$. Let $M$ be the vector space over $F$ with $x_{1}, \ldots, x_{2 R}$ as basis and let $a=a(x, y)$ be a skew-symmetric bilinear form on $M$ with $a\left(x_{i}, x_{j}\right)=\alpha_{i j}=-\alpha_{j i}$ whose rank is equal to $2 R$.

Define $A^{+}$by $A^{+}=F 1+N^{+}$where $N^{+}=F\left[x_{1}, \ldots, x_{2 R}\right]$, with $x_{i}^{p}=0, i=1, \ldots, 2 R$. For convenience write $x_{i}^{0}=1, i=1, \ldots, 2 R$. That is, $A^{+}$is a commutative associative truncated polynomial algebra with nilpotent generators. Now define $A=F 1+N$ to be the same vector space as $A^{+}$with generator products defined by $x_{i} x_{j}=\alpha_{i j} 1$ $+w_{i j}$, with $w_{i j}=2 x_{i} \cdot x_{j}-w_{j i}$ in $N$ for $i<j$. Further, define

$$
f g=f \cdot g+\frac{1}{2} \sum_{i, j=1}^{2 R} \frac{\partial f}{\partial x_{i}} \cdot \frac{\partial g}{\partial x_{j}} \cdot\left[x_{i}, x_{j}\right]
$$

for any elements $f$ and $g$ of $A$.

We note here that Kokoris [3, Theorems 5 and 6] has proved that the above conditions and construction of $A$ are sufficient for $A$ to be a simple nodal noncommutative Jordan algebra in the class $K$.

Finally, complete the definition of $A$ by setting $w_{23}=w_{32}, w_{13}=w_{31}$, and choosing $w_{12}$ such that $\left[w_{12}, x_{3}\right] \neq 2\left(\alpha_{13} x_{2}+\alpha_{23} x_{1}\right)$.

We shall show that $A$ as defined above cannot be Lie-admissible, since the Jacobi identity in $A^{-}$is not satisfied. For consider the quantity

$$
\begin{aligned}
J & =\left[\left[x_{1}, x_{2}\right], x_{3}\right]+\left[\left[x_{2}, x_{3}\right], x_{1}\right]+\left[\left[x_{3}, x_{1}\right], x_{2}\right] \\
& =\left[w_{12}-w_{21}, x_{3}\right]=2\left[w_{12}-x_{1} \cdot x_{2}, x_{3}\right] .
\end{aligned}
$$

It has been previously noted that

Thus

$$
\left[x_{1} \cdot x_{2}, x_{3}\right]=\left[x_{1}, x_{3}\right] \cdot x_{2}+x_{1} \cdot\left[x_{2}, x_{3}\right]=2\left(\alpha_{13} x_{2}+\alpha_{23} x_{1}\right) .
$$

$$
J=2\left[w_{12}, x_{3}\right]-4\left(\alpha_{13} x_{2}+\alpha_{23} x_{1}\right) \neq 0
$$

and $A^{-}$is not a Lie algebra.

Now suppose that there exists a set of generators $y_{1}, \ldots, y_{2 R}$ for $A$ satisfying the relations (1). By Theorem 2, we must have $A$ Lie-admissible, which is a contradiction. Theorem 3 is proved.

\section{REFERENCES}

1. A. A. Albert, On commutative power-associative algebras of degree 2, Trans. Amer. Math. Soc. 74 (1953), 323-343.

2. L. A. Kokoris, Simple nodal noncommutative Jordan algebras, Proc. Amer. Math. Soc. 9 (1958), 652-654. 
3. - Nodal noncommutative Jordan algebras, Canad. J. Math. 12 (1960), 488-492.

4. R. H. Oehmke, Nodal noncommutative Jordan algebras, Trans. Amer. Math. Soc. 112 (1964), 416-431.

5. R. D. Schafer, Noncommutative Jordan algebras of characteristic 0, Proc. Amer. Math. Soc. 6 (1955), 472-475.

6. - On noncommutative Jordan algebras, Proc. Amer. Math. Soc. 9 (1958), 110-117.

7. - Nodal noncommutative Jordan algebras and simple Lie algebras of characteristic $p$,

Trans. Amer. Math. Soc. 94 (1960), 310-326.

IlLinOIS INSTITUTE OF TeCHNOLOGY,

Chicago, Illinois 